\title{
Optical Fluorescence Detected from X-ray Irradiated Liquid Water
}

\author{
Andreas Hans, ${ }^{*}{ }^{\dagger}$ Christian Ozga, ${ }^{\dagger}$ Robert Seidel, ${ }^{\ddagger}$ Philipp Schmidt, ${ }^{\dagger}$ \\ Timo Ueltzhöffer, ${ }^{\dagger}$ Xaver Holzapfel, ${ }^{\dagger}$ Philip Wenzel, ${ }^{\dagger}$ Philipp Reiß, ${ }^{\dagger}$ \\ Marvin N. Pohl,,$\uparrow$ Isaak Unger, ${ }^{\S}$ Emad F. Aziz,,,$+\|$,$\| Arno Ehresmann, { }^{\dagger}$ \\ Petr Slavíček, ${ }^{\perp}$ Bernd Winter, ${ }^{\ddagger}$ and André Knie*,† \\ $\dagger$ University of Kassel, Department of Physics, Heinrich-Plett-Strasse 40, D-34132 Kassel, \\ Germany \\ $\ddagger$ Helmholtz-Zentrum Berlin für Materialien und Energie, Methods for Material \\ Development, Albert-Einstein-Strasse 15, D-12489 Berlin, Germany \\ IDepartment of Physics, Free University Berlin, Arnimallee 14, D-14195 Berlin, Germany \\ $\S$ Department of Physics and Astronomy, Uppsala University, Box 516, SE-75120 Uppsala, \\ Sweden \\ ||School of Chemistry, Monash University, Clayton Campus, Victoria 3800, Australia \\ $\perp$ Department of Physical Chemistry, University of Chemistry and Technology Prague, \\ Technická 5, 16628 Prague, Czech Republic \\ E-mail: hans@physik.uni-kassel.de; knie@physik.uni-kassel.de
}




\begin{abstract}
Despite its importance, the structure and dynamics of liquid water are still poorly understood in many apsects. Here, we report on the observation of optical fluorescence upon soft X-ray irradiation of liquid water. Detection of spectrally resolved fluorescence was achieved by a combination of the liquid microjet technique and fluorescence spectroscopy. We observe a genuine liquid-phase fluorescence manifested by a broad emission band in the 170 to $340 \mathrm{~nm}(4-7 \mathrm{eV})$ photon wavelength range. In addition, another narrower emission near $300 \mathrm{~nm}$ can be assigned to the fluorescence of $\mathrm{OH}(\mathrm{A}$ state) in the gas phase, the emitting species being formed by Auger electrons escaping from liquid water. We argue that the newly observed broad-band emission of liquid water is relevant in search of extraterrestrial life, and we also envision the observed electronejection mechanism to find application for exploring solutes at liquid-vapor interfaces.
\end{abstract}

\title{
Introduction
}

Despite water's importance, many of its peculiar properties still remain poorly understood on a molecular level. ${ }^{1}$ For instance, its exact role in the function of biological systems is subject of intense current research. ${ }^{2}$ Tackling the complexity of the molecular structure of water with its fluxional hydrogen-bonding network, involving rapid bond-breaking and bond-making dynamics, is an ongoing experimental and theoretical challenge. ${ }^{3-10}$

The dynamical electronic and sometimes even nuclear dynamics initiated by X-ray irradiation can be traced, e.g. by electron spectroscopy. Yet the application to the liquid phase has been only possible after the recent development of the liquid microjet technique, enabling high-vacuum electron-spectroscopy experiments from highly volatile liquids. ${ }^{3,11-15}$

Due to its omnipresence and unique properties, liquid water is one of the key players in the development and evolution of life on earth and therefore regarded a main prerequisite for the habitability of celestial bodies, i.e. for the existence of extraterrestrial life. ${ }^{16,17}$ Optical fluorescence emitted from molecular water, including its atomic and molecular fragments, 
is of large astrophysical importance. Identification of water-specific emissions in the optical region can be used for remote detection of water on extraterrestrial objects. The presence of constituents of the water molecule can either serve as a production source for water itself, or they are indicator for the occurrence of water, decomposed by extreme UV or X-ray radiation. ${ }^{19}$ The UV/visible fluorescence spectrum of the gas-phase water molecule, with excited or ionized valence or inner orbitals, has been studied in detail ${ }^{20-22}$ and is dominated by the emission of water fragments, with a minor contribution from excited $\mathrm{H}_{2} \mathrm{O}^{+}$. The most prominent emission features in the $150-600 \mathrm{~nm}$ region are the Balmer transitions of the neutral hydrogen atom, ${ }^{23,24}$ and the $\mathrm{A} \rightarrow \mathrm{X}$ transitions of the hydroxyl radical, $\mathrm{OH}$, at $290 \mathrm{~nm}$ and $310 \mathrm{~nm} .{ }^{25}$ This characteristic fluorescence indeed plays an important role the search of $\mathrm{OH}$ in planetary atmospheres. ${ }^{26}$ Also luminescence of solid water in various ice phases has been subject to intense investigations. Luminescence was observed following UV irradiation and high-energy electron excitation. ${ }^{27-32}$ However, optical emission spectra which would correspond to the emissions observed here have not been reported to date. A first such report on core-level ionization-induced optical emission from liquid water is presented here.

The application of X-rays, as well as the detection of fluorescence in the deep UV, requires high vacuum conditions because of the large absorption cross section for most atmospheric gases in the relevant energy range. Due to these difficulties, only few works on luminescence from liquid water at all have been reported. Evidence for luminescence in the UV emission range has been found in early works. ${ }^{33}$ In particular, sonoluminescence experiments revealed broad emission bands between $200 \mathrm{~nm}$ and about $500 \mathrm{~nm}$, which have been assigned to the $\mathrm{OH}$ radical, ${ }^{34,35}$ resulting from water fragmentation. In the present work, we have combined the liquid microjet technique ${ }^{11,36,37}$ with dispersed fluorescence detection, ${ }^{22,38-42}$ which enables the investigation of photon-induced luminescence with an unprecedented sensitivity. 


\section{Methods}

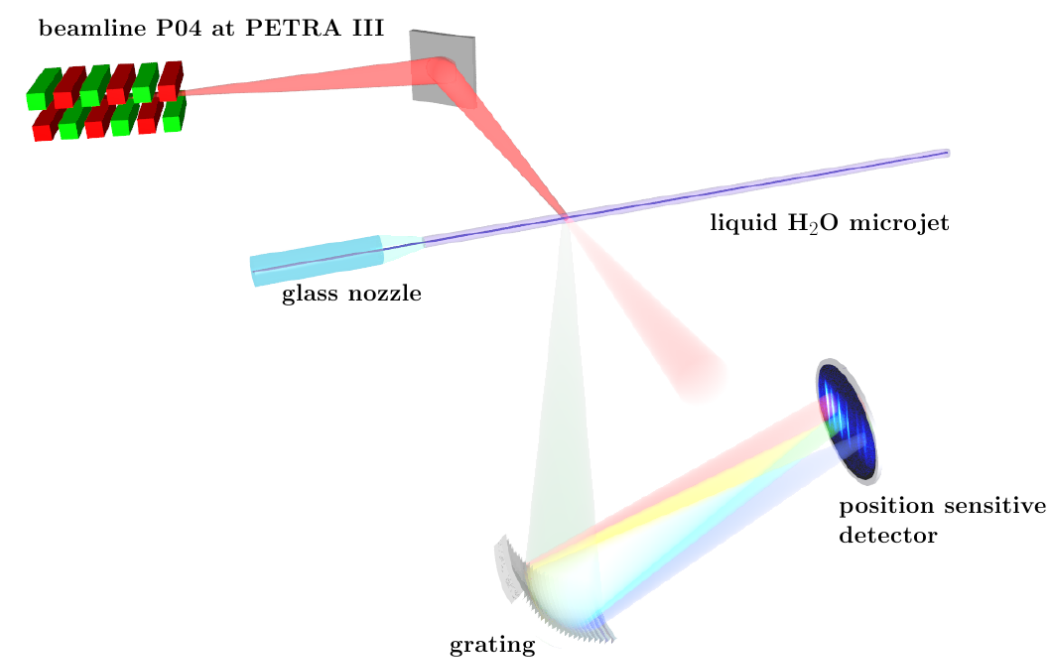

Figure 1: Sketch of the experimental setup. A liquid water jet of $24 \mu \mathrm{m}$ diameter is crossed with the soft X-ray light from the P04 beamline of PETRA III. The entrance path into the photon spectrometer is perpendicular to the plane spanned by jet and synchrotron radiation. The dispersed fluorescence is detected by a position sensitive single photon detector. Dimensions are not to scale.

The experiment was performed at the P04 beamline of the synchrotron-light facility PETRA III at DESY in Hamburg, ${ }^{43}$ providing a photon beam with a micro-focus of $5 \times 10 \mu \mathrm{m}^{2}$. The synchrotron radiation was crossed with a liquid waterjet of $24 \mu \mathrm{m}$ diameter; the jet was operated at room temperature. The experimental scheme is sketched in Figure 1. Measurements were performed at $10^{-4}$ mbar pressure inside the interaction chamber, achieved by a combination of turbomolecular pumps and cold traps (at liquid nitrogen temperature). A $1 \mathrm{~mm}$ entrance slit to a photon spectrometer was mounted at the axis perpendicular to the plane spanned by the synchrotron beam and the liquid jet. To disperse the fluorescence, a 1 m normal incidence MCPHERSON spectrometer was used, equipped with an aluminumcoated grating with 600 lines per millimeter. The spectrally resolved photons were detected with a position-sensitive detector, consisting of a stack of micro-channel plates (MCPs) and a bialkali-coated photocathode. 
Here, the setup is also used for time-resolved measurements, where the time at which the photons are emitted (with respect to the ionizing X-ray pulse) is evaluated. For that the PETRA III storage ring bunch marker is used to trigger a time-to-digital converter (TDC) which is stopped by the MCP pulse generated by each single photon hitting the detector. The obtained event times (with respect to the bunch marker) are collected and mapped onto a histogram. The lifetime of excited states decaying radiatively can be extracted directly from this histogram by fitting an exponential decay starting at the time of the bunch marker. The lower limit of the time resolution of this method is given by the synchrotron bunch width and the TDC channel width, and is about $150 \mathrm{ps}$. The upper limit corresponds to the temporal distance between two electron bunches, which was $140 \mathrm{~ns}$ in the present experiment. However, a fitting procedure considering fluorescence signal contributions due to consecutive bunches (arriving at multiples of $140 \mathrm{~ns}$ ) can be used to measure even longer lifetimes, up to $1 \mu \mathrm{s}$ in the present work.

\section{Results \& Discussion}

Figure 2 shows two optical fluorescence spectra obtained upon excitation of the liquid waterjet by soft X-rays. The spectrum in Figure 2A was obtained for a photon energy well above the oxygen 1s ionization energy of liquid water $\left(538.1 \mathrm{eV}^{44}\right)$ using $600 \mathrm{eV}$, and in Figure 2B the X-ray energy was $530 \mathrm{eV}$ which is just below the $\mathrm{O}$ 1s absorption edge $\left(533 \mathrm{eV}^{45}\right)$. The spectrum in Figure 2A exhibits three distinct features: Sharp atomic emission lines (green, label I), molecular emission bands (red, label II), and a very broad, hitherto unobserved emission band (gray, label III) from approximately 170 to $340 \mathrm{~nm}$. Feature III does not show significant differences for the different exciting-photon energies within the present experimental resolution and statistics. The double-line emission I is identified as the $2 \mathrm{~s}^{2} 2 \mathrm{p}^{2}\left({ }^{1} \mathrm{D}\right) 3 \mathrm{~d}^{2} \mathrm{P}_{1 / 2,3 / 2} \rightarrow 2 \mathrm{~s}^{2} 2 \mathrm{p}^{2}\left({ }^{3} \mathrm{P}\right) 3 \mathrm{p}^{2} \mathrm{D}_{3 / 2,5 / 2}$ transitions of the free $\mathrm{O}^{+}$fragment. ${ }^{46}$ Emissions II and III arise, however, from the liquid phase, and will be detailed next. 


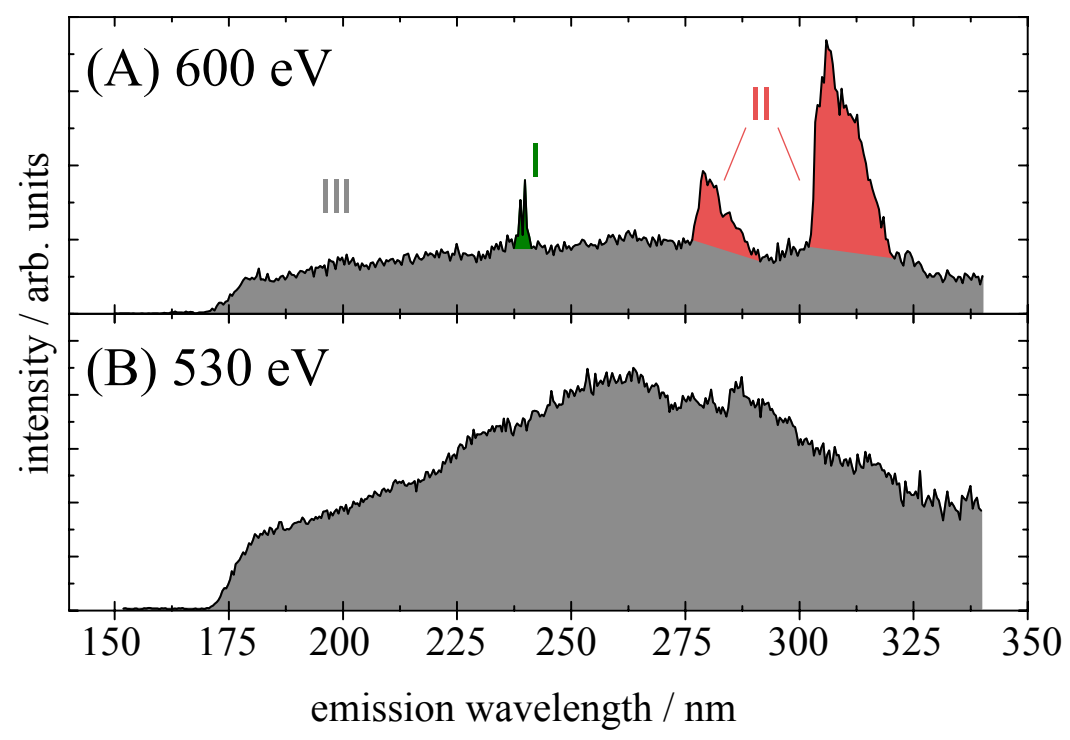

Figure 2: Optical emission spectra of a liquid water jet. (A) Exciting-photon energy $600 \mathrm{eV}$. (B) Exciting-photon energy $530 \mathrm{eV}$. Features I, II and III are labelled according to the processes discussed in the text.

To elucidate the nature of the emitting species and how they form, the excitation function of the respective signal intensity is measured in the vicinity of the $\mathrm{O} 1$ s edge, i.e., the excitation-photon energy is varied stepwise across the $\mathrm{O} 1 \mathrm{~s}$ resonances, and the intensity of each emission feature is determined. The results are shown in Figure 3. Statistics of the excitation function of feature I (Figure 3A, gray dotted curve) are poor owing to the very low count rate compared to features II and III. However, the overall shape resembles the well known excitation function of emission from gas-phase water ${ }^{21,22}$ and three characteristic peaks at about $534.0 \mathrm{eV}, 535.9 \mathrm{eV}$, and $537.1 \mathrm{eV}$ can clearly be assigned to inner-shell excitations in molecular water. ${ }^{45}$ Feature I must hence be arising from the excitation of gas-phase water molecules at very close distance from the liquid jet. This is illustrated in Figure 4.

The shape of the excitation function for feature II (red curve in Figure 3A) reproduces the X-ray absorption (XA) spectrum of liquid water, ${ }^{5,6}$ which identifies this emission feature as being associated with the initial O 1s excitation of liquid water. Surprisingly though, the emission wavelengths and spectral structure agree with the emission of gas-phase $\mathrm{OH}$ in the 


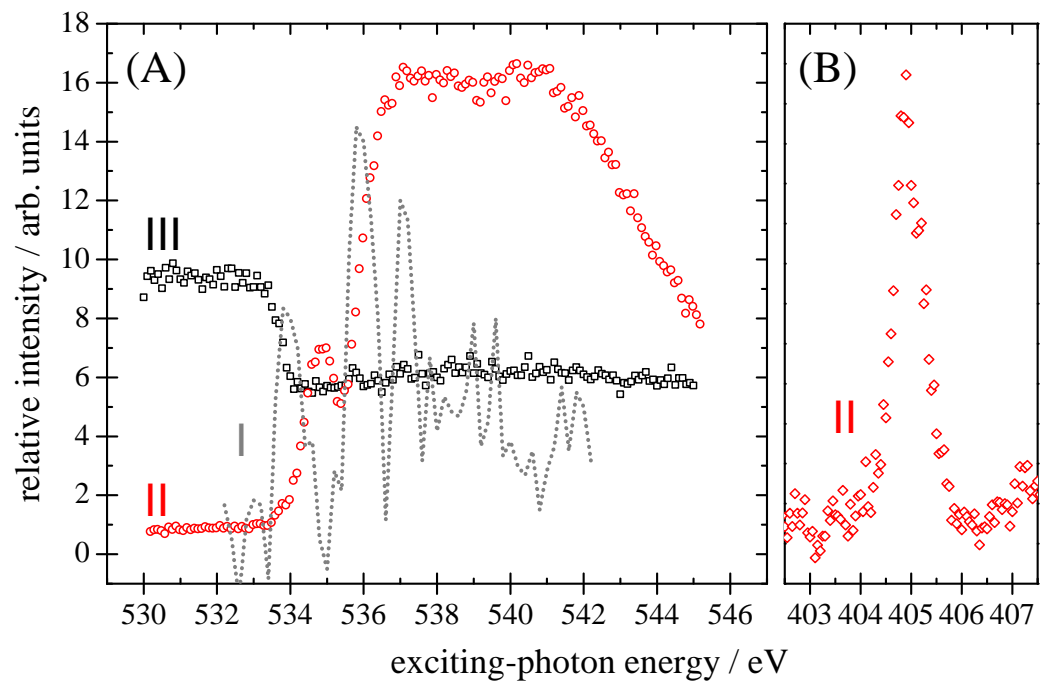

Figure 3: (A) Excitation functions of features I (dotted line), II (red circles) and III (black squares) across the oxygen 1s edge for a neat water sample. The relative intensities are derived from the integrated intensity of the full fluorescence range investigated (150-340 nm) and valid for features II and III. The intensity of feature I is on arbitrary scale and not scaled relative to features II and III. (B) Excitation function of feature II across the nitrogen 1s- $\pi^{*}$ resonance of a 1 molar sodium nitrate $\left(\mathrm{NaNO}_{3}\right)$ solution.

A state, ${ }^{25}$ implying that feature II arises from a process induced by inner-shell excitation of liquid water which then leads to excited $\mathrm{OH}$ (gas), located in the very close vicinity to the liquid-vacuum interface. The suggested mechanism for that is electron-impact excitation of interfacial gas-phase $\mathrm{H}_{2} \mathrm{O}$ largely by Auger electrons (of approximately $500 \mathrm{eV}$ kinetic energy) emitted from core-hole excited liquid water; the process is also illustrated in Figure 4. At O 1s resonant excitation the Auger electron yield is known to be very large, $>88 \%$ has been estimated for core-level ionization. ${ }^{14}$ The red curve (II) in Figure 3A can then be attributed to the optical fluorescence of gas-phase $\mathrm{OH}$ in the $\mathrm{A}$ state created by the dissociation of $\mathrm{H}_{2} \mathrm{O}$ (gas) upon impact of $500 \mathrm{eV}$ Auger electrons. These leading edge Auger electrons from liquid water ${ }^{44}$ can be regarded as a broadband excitation. ${ }^{47}$ Note that for photon energies just below the $\mathrm{O}$ 1s edge photoelectrons from valence levels have about the same kinetic energy as these Auger electrons. However, the absorption cross section below the edge is that low, that a vast majority of photons is absorbed in the bulk of the liquid jet, while feature II 
is a pure interface effect. That explains why feature II is not present in the spectrum in Figure 2B. Above the 1s edge, most photons are already absorbed at the jet surface due to the large absorption cross section, and the intensity of feature II is tremendously enhanced.

To confirm our interpretation, we also measured the excitation function of feature II for the nitrogen $1 \mathrm{~s}-\pi^{*}$ resonance regime at about $405 \mathrm{eV}$ of a 1 molar sodium nitrate $\left(\mathrm{NaNO}_{3}\right)$ solution, which is shown in Figure 3B. This excitation function well agrees with recent total electron yield X-ray absorption spectra. ${ }^{48}$ We therefore conclude that also electrons from the solute will considerably scatter on their way through the gas halo surrounding the liquid jet, thereby electronically exciting water molecules. Processes which are initiated by Auger electrons originating from the liquid phase, and subsequently leading to highly excited molecules right above the liquid surface have not been considered before to our knowledge. The present study therefore identifies a thus far unnoticed relaxation pathway that uniquely couples both components of the liquid-gas interface. This process might find applications in the characterization of solutes and liquid-vapor interfaces, as the intensity of the signal is proportional to the number of electrons escaping from the surface and the spectrum reflects the interfacial composition.

The broad-band emission III in the present shape has not been reported before for any water-derived atomic or diatomic gas-phase species, and must hence be a signature of waterderived species in solution; compare illustration in Figure 4. Sonoluminescence studies have suggested that hydrated $\mathrm{OH}$ in the $\mathrm{A}$ and $\mathrm{C}$ states exhibits an emission in a similar spectral range, with an onset near $200 \mathrm{~nm}$ and extending to above $400 \mathrm{~nm} \cdot{ }^{34,35}$ The spectrum recorded in our work differs however in shape and width, and there seems to be no obvious correspondence to the sonoluminescence spectra. Note that the low-energy onset of band III in the emission spectrum cannot be accurately determined from the present data because for all wavelengths above $340 \mathrm{~nm}$ emission contributions from first and second order of the spectrometer overlap, and cannot be separated with the present setup. The steep high-energy cutoff at about $170 \mathrm{~nm}$ corresponds to the onset of UV absorption of liquid water, ${ }^{49}$ i. e. 


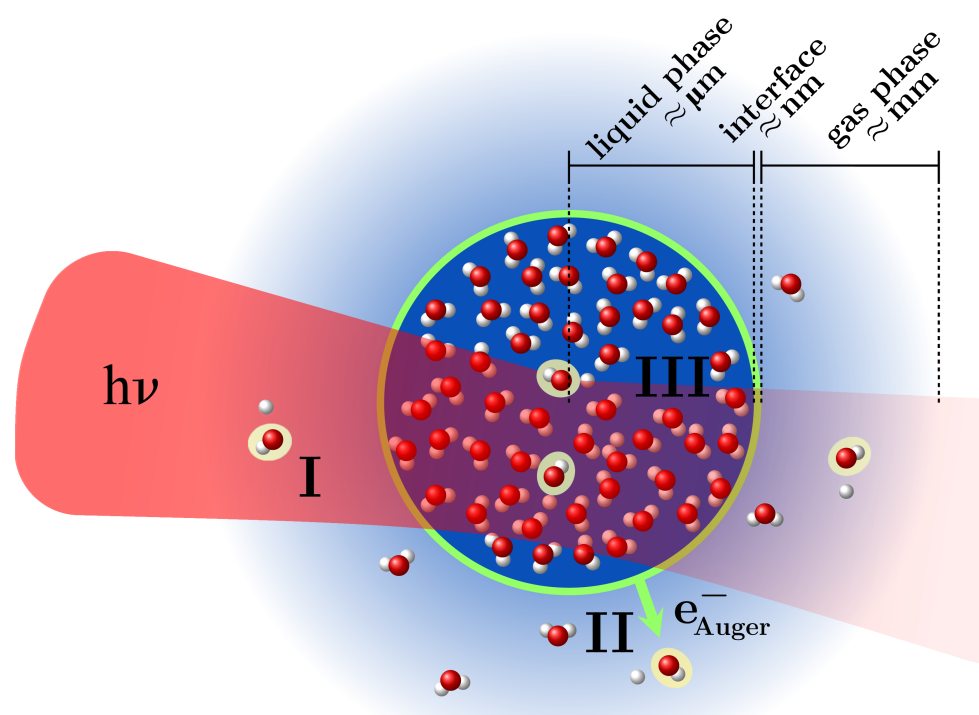

Figure 4: Close-up of the interaction region and illustration of the different processes leading to the emission features I (emission upon excitation of gas-phase water molecules by synchrotron radiation), II (emission upon excitation of gas-phase water molecules by Auger electrons from the gas-liquid interface), and III (emission from a transient species in liquidphase water).

water is opaque for shorter wavelengths. The full width of this emission feature is therefore not accessible owing to the intrinsic absorption properties of water. Unlike for feature II, the excitation function of feature III is flat below the $\mathrm{O}$ 1s edge, i.e., for photon energies less than $533 \mathrm{eV}$. Then, at the edge the cross section exhibits a sudden jump to a lower, again constant signal level extending to larger photon energies. The only process known to occur for photon energies in the range below $533 \mathrm{eV}$ is the ionization of water valence orbitals, with binding energies between 11 and $32 \mathrm{eV} .{ }^{50}$ The drop of signal III at the $\mathrm{O}$ 1s edge is then assigned to a saturation effect. For a non-transparent liquid jet, assuming a constant photon absorption rate close to $100 \%$, the total number of valence ionization events is reduced as soon as inner-shell excitation becomes possible as the cross section of resonant inner-shell photon absorption is considerably larger than the cross section for valence ionization in this energy range.

In an attempt to obtain further insight into the nature of the processes responsible for the III-band emission we have measured the lifetimes of the respective process associated with 
features II and III. The decay time for the emission of feature III is observed to be at the lower experimental temporal resolution, and we can only provide an upper limit of approximately 150 ps. No radiative state exhibiting such short fluorescence lifetime of any water-dissociation product has been reported. ${ }^{20}$ The fastest reported fluorescence decay of a water fragment is $1.6 \mathrm{~ns}$ for $\mathrm{Ly}_{\alpha}$ emission from neutral hydrogen atoms, which is yet an order of magnitude slower than observed here for the III-emission. Most likely the observed 150 ps decay time is rather a measure of the survival time of a particular excited but transient species until it decays by competing non-radiative processes. The initial excited species will strongly react with the solvent molecules, and thus be consumed faster than the actual, and experimentally not accessible radiative lifetime. The large width of the fluorescence spectrum cannot be explained by emission of a single species even if inhomogeneous broadening were taken into account. Hence, the observed signal must be attributed to the simultaneous emission from a larger number of excited oxygenic species with very short lifetimes, most likely $\mathrm{OH}$ radicals in various excited states. This leads to large variation of hydration structures, and hence to considerable spectral broadening and peak shifts. In contrast to the short process lifetime of feature III, the lifetime of feature II, measured at $538 \mathrm{eV}$, is determined to be 580 ns, i.e., orders of magnitude larger. Within the experimental uncertainty $( \pm 20 \%)$, this value can be assigned to the $\mathrm{A}$ state of $\mathrm{OH}$ in the gas phase, reported at $700 \mathrm{~ns} .{ }^{51}$ Note that the timeresolved measurement enables an efficient discrimination between features II and III, which can be used in future studies to separately investigate the underlying mechanisms. That is, if a proper time gating is used, both features/mechanisms can be detected separately, even if overlapping in excitation or emission energy.

\section{Conclusion}

In conclusion, we were able to detect and analyze the weak optical fluorescence signal from $\mathrm{X}$-ray irradiated liquid water. This was possible using an experimental setup which com- 
bines the liquid microjet with photon-induced fluorescence spectrometry. Our measurements have identified two previously undetected optical fluorescence features connected to X-ray irradiated liquid water. One is a genuine fluorescence where photons are emitted from the liquid phase. Extending over a large energy range between 170 and $340 \mathrm{~nm}$, this emission is argued to have contributions from many transient species with very short lifetimes, occurring upon fragmentation of water molecules in the liquid phase. The other fluorescence contribution is initiated by the excitation of gas-phase water molecules by (Auger) electrons emitted from liquid water, transferred across the liquid-vapor boundary. The discovered broad liquid-phase emission band is regarded a new spectroscopic fingerprint of electronically excited liquid water, which can be utilized in the search for water in liquid phase in interstellar space. Both features can be also used for the investigation of the dynamics of liquid water both on the surface and in the bulk of the liquid, and are believed to find several future applications. We particularly envision the Auger electron induced excitation across the interface as a potential novel tool for the characterization of aqueous-solution-air interfaces. The technique presented here can also contribute to advances in the emerging technology in X-ray excited luminescence, e.g. in the field of biomedical imaging. ${ }^{52,53}$

\section{Acknowledgement}

This work was supported by the State of Hesse Initiative for the Development of Scientific and Economic Excellence (LOEWE) within the focus-project Electron Dynamics of Chiral Systems (ELCH), and by the Deutsche Forschungsgemeinschaft (Forschergruppe FOR 1789).

P.S. is supported by the Czech Science Foundation (13-34168S). We acknowledge the P04 beamline team and the DESY staff for excellent support during the experiments.

\section{References}

(1) Ball, P. Water - an Anduring Mystery. Nature 2008, 452, 291-292. 
(2) Maréchal, Y. Water and Biomolecules: An Introduction. J. Mol. Struct. 2004, 700, $207-210$.

(3) Slavíček, P.; Winter, B.; Cederbaum, L. S.; Kryzhevoi, N. V. Proton-Transfer Mediated Enhancement of Nonlocal Electronic Relaxation Processes in X-ray Irradiated Liquid Water. J. Am. Chem. Soc. 2014, 136, 18170-18176.

(4) Skinner, L. B.; Huang, C.; Schlesinger, D.; Pettersson, L. G. M.; Nilsson, A.; Benmore, C. J. Benchmark Oxygen-Oxygen Pair-Distribution Function of Ambient Water from X-ray Diffraction Measurements with a Wide Q-Range. J. Chem. Phys. 2013, 138, 074506.

(5) Wernet, P.; Nordlund, D.; Bergmann, U.; Cavalleri, M.; Odelius, M.; Ogasawara, H.; Näslund, L. A.; Hirsch, T. K.; Ojamäe, L.; Glatzel, P.; Pettersson, L. G. M.; Nilsson, A. The Structure of the First Coordination Shell in Liquid Water. Science 2004, 304, 995999.

(6) Smith, J. D.; Cappa, C. D.; Wilson, K. R.; Messer, B. M.; Cohen, R. C.; Saykally, R. J. Energetics of Hydrogen Bond Network Rearrangements in Liquid Water. Science 2004, 306, 851-853.

(7) Huang, C.; Wikfeldt, K. T.; Tokushima, T.; Nordlund, D.; Harada, Y.; Bergmann, U.; Niebuhr, M.; Weiss, T. M.; Horikawa, Y.; Leetmaa, M.; et al. The Inhomogeneous Structure of Water at Ambient Conditions. Proc. Natl. Acad. Sci. U. S. A. 2009, 106, $15214-15218$.

(8) Clark, N. I. G.; Cappa, C. D.; Smith, J. D.; Saykally, R. J.; Head-Gordon, T. The Structure of Ambient Water. Mol. Phys. 2010, 108, 1415-1433.

(9) Henchman, R. H.; Cockram, S. J. Water's Non-Tetrahedral Side. Faraday Discuss. 2013, 167, 529-550. 
(10) Kühne, T. D.; Khaliullin, R. Z. Electronic Signature of the Instantaneous Asymmetry in the First Coordination Shell of Liquid Water. Nat. Commun. 2013, 4, 1450.

(11) Winter, B. Liquid Microjet for Photoelectron Spectroscopy. Nucl. Instrum. Methods Phys. Res., Sect. A 2009, 601, 139-150.

(12) Nilsson, A.; Pettersson, L. G. M. Perspective on the Structure of Liquid Water. Chem. Phys. 2011, 389, 1-34.

(13) Thürmer, S.; Ončák, M.; Ottosson, N.; Seidel, R.; Hergenhahn, U.; Bradforth, S. E.; Slavičček, P.; Winter, B. On the Nature and Origin of Dicationic, Charge-Separated Species Formed in Liquid Water on X-ray Irradiation. Nat. Chem. 2013, 5, 590-596.

(14) Slavíček, P.; Kryzhevoi, N. V.; Aziz, E. F.; Winter, B. Relaxation Processes in Aqueous Systems upon X-ray Ionization: Entanglement of Electronic and Nuclear Dynamics. $J$. Phys. Chem. Lett. 2016, 7, 234-243.

(15) Fransson, T.; Harada, Y.; Kosugi, N.; Besley, N. A.; Winter, B.; Rehr, J. J.; Pettersson, L. G. M.; Nilsson, A. X-ray and Electron Spectroscopy of Water. Chem. Rev. 2016, $116,7551-7569$.

(16) Kasting, J. F.; Catling, D. Evolution of a Habitable Planet. Annu. Rev. Astron. Astrophys. 2003, 41, 429-463.

(17) Kasting, J. F.; Harman, C. E. Extrasolar Planets: Inner Edge of the Habitable Zone. Nature 2013, 504, 221-223.

(18) Thürmer, S.; Seidel, R.; Faubel, M.; Eberhardt, W.; Hemminger, J. C.; Bradforth, S. E.; Winter, B. Photoelectron Angular Distributions from Liquid Water: Effects of Electron Scattering. Phys. Rev. Lett. 2013, 111, 173005.

(19) Johnson, R. E.; Quickenden, T. I. Photolysis and Radiolysis of Water Ice on Outer Solar System Bodies. J. Geophys. Res. 1997, 102, 10985-10996. 
(20) Dutuit, O.; Tabche-Fouhaile, A.; Nenner, I.; Frohlich, H.; Guyon, P. M. Photodissociation Processes of Water Vapor Below and Above the Ionization Potential. J. Chem. Phys. 1985, 83, 584-596.

(21) Kivimäki, A.; Coreno, M.; Richter, R.; Ruiz Álvarez, J.; Melero García, E.; de Simone, M.; Feyer, V.; Vall-llosera, G.; Prince, K. C. Fluorescence Emission Following Core Excitations in the Water Molecule. J. Phys. B: At., Mol. Opt. Phys. 2006, 39, 1101-1112.

(22) Hans, A.; Knie, A.; Schmidt, Ph.; Ben Ltaief, L.; Ozga, C.; Reiß, P.; Huckfeldt, H.; Förstel, M.; Hergenhahn, U.; Ehresmann, A. Lyman-Series Emission after Valence and Core Excitation of Water Vapor. Phys. Rev. A 2015, 92, 032511.

(23) Melero García, E.; Kivimäki, A.; Pettersson, L. G. M.; Alvarez Ruiz, J.; Coreno, M.; de Simone, M.; Richter, R.; Prince, K. C. Fluorescence Emission of Excited Hydrogen Atoms after Core Excitation of Water Vapor. Phys. Rev. Lett. 2006, 96, 063003.

(24) Kato, M.; Odagiri, T.; Kodama, K.; Murata, M.; Kameta, K.; Kouchi, N. Doubly Excited States of Water in the Inner Valence Range. J. Phys. B: At., Mol. Opt. Phys. 2004, 37, 3127-3148.

(25) Zanganeh, A. H.; Fillion, J. H.; Ruiz, J.; Castillejo, M.; Lemaire, J. L.; Shafizadeh, N.; Rostas, F. Photodissociation of $\mathrm{H}_{2} \mathrm{O}$ and $\mathrm{D}_{2} \mathrm{O}$ below $132 \mathrm{~nm}$. J. Chem. Phys. 2000, 112, 5660-5671.

(26) Shemansky, D. E.; Matheson, P.; Hall, D. T.; Hu, H.-Y.; Tripp, T. M. Detection of the Hydroxyl Radical in the Saturn Magnetosphere. Nature 1993, 363, 329-331.

(27) Langford, V. S.; McKinley, A. J.; Quickenden, T. I. Luminescent Photoproducts in UV-Irradiated Ice. Acc. Chem. Res. 2000, 33, 665-671. 
(28) Selby, B. J.; Quickenden, T. I.; Freeman, C. G. Isotopic Effects on the Time-Dependence of $420 \mathrm{~nm}$ Ice Luminescence Excited by UV Light. Kinet. Catal. 2006, 47, 686-698.

(29) Quickenden, T. I.; Green, T. A.; Lennon, D. Luminescence from UV-Irradiated Amorphous $\mathrm{H}_{2} \mathrm{O}$ Ice. J. Phys. Chem. 1996, 100, 16801-16807.

(30) Freeman, C. G.; Quickenden, T. I.; Litjens, R. A. J.; Sangster, D. F. Visible and Ultraviolet Emission from Pulse Irradiated Amorphous and Polycrystalline $\mathrm{H}_{2} \mathrm{O}$ Ice. $J$. Chem. Phys. 1984, 81, 5252-5254.

(31) Matich, A. J.; Bakker, M. G.; Lennon, D.; Quickenden, T. I.; Freeman, C. G. $\mathrm{O}_{2}$ Luminescence from UV-Excited $\mathrm{H}_{2} \mathrm{O}$ and $\mathrm{D}_{2} \mathrm{O}$ Ices. J. Phys. Chem. 1993, 97, 1053910553.

(32) Miyazaki, T.; Nagasaka, S.; Kamiya, Y.; Tanimura, K. Formation of Excited OH Radicals in High-Energy-Electron-Irradiated Ice at Very Low Temperature. J. Phys. Chem. 1993, 97, 10715-10719.

(33) Quickenden, .T. I.; Arvin, J. A.; Sangster, D. F. A Search for Non-Čerenkov UV Emission from Liquid Water Irradiated with Pulsed Electrons. J. Chem. Phys. 1980, 73, $3632-3634$.

(34) Didenko, Y. T.; Pugach, S. P. Spectra of Water Sonoluminescence. J. Phys. Chem. 1994, 98, 9742-9749.

(35) Pflieger, R.; Brau, H.-P.; Nikitenko, S. I. Sonoluminescence from $\mathrm{OH}\left(\mathrm{C}^{2} \Sigma^{+}\right)$and $\mathrm{OH}\left(\mathrm{A}^{2} \Sigma^{+}\right)$Radicals in Water: Evidence for Plasma Formating during Multibubble Cavitation. Chem. - A Eur. J. 2010, 16, 11801-11803.

(36) Winter, B.; Faubel, M. Photoemission from Liquid Aqueous Solutions. Chem. Rev. 2006, 106, 1176-1211. 
(37) Seidel, R.; Thürmer, S.; Winter, B. Photoelectron Spectroscopy Meets Aqueous Solution. J. Phys. Chem. Lett. 2011, 2, 633-641.

(38) Liebel, H.; Lauer, S.; Vollweiler, F.; Müller-Albrecht, R.; Ehresmann, A.; Schmoranzer, H.; Mentzel, G.; Schartner, K.-H.; Wilhelmi, O. Neutral Photodissociation of $\mathrm{O}_{2}$ Rydberg States Accompanied by Changes of the Rydberg Electron's Quantum Numbers $n$ and $l$. Phys. Lett. A 2000, 267, 357-369.

(39) Zimmermann, B.; Wilhelmi, O.; Schartner, K.-H.; Vollweiler, F.; Liebel, H.; Ehresmann, A.; Lauer, S.; Schmoranzer, H.; Lagutin, B. M.; Petrov, I. D.; et al. Alignment of Ions after Autoionization Decay of Atomic Resonances: II. The $3 \mathrm{~d}_{5 / 2}^{9} 5 \mathrm{p}(J=1)$ Resonance in Kr. J. Phys. B: At., Mol. Opt. Phys. 2000, 33, 2467-2478.

(40) Demekhin, P. V.; Petrov, I. D.; Sukhorukov, V. L.; Kielich, W.; Knie, A.; Schmoranzer, H.; Ehresmann, A. Symmetry-Forbidden Electronic State Interference Observed in Angularly Resolved $\mathrm{NO}^{+}\left(A^{1} \Pi\right)$ Deexcitation Spectra of the $\mathrm{N}^{*} \mathrm{O}\left(2 \sigma^{-1} 2 \pi^{2}\right)$ Resonance. Phys. Rev. Lett. 2010, 104, 243001.

(41) Knie, A.; Ilchen, M.; Schmidt, Ph.; Reiß, P.; Ozga, C.; Kambs, B.; Hans, A.; Müglich, N.; Galitskiy, S. A.; Glaser, L.; et al. Angle-Resolved Study of Resonant Auger Decay and Fluorescence Emission Processes after Core Excitations of the Terminal and Central Nitrogen Atoms in $\mathrm{N}_{2}$ O. Phys. Rev. A 2014, 90, 013416.

(42) Knie, A.; Hans, A.; Förstel, M.; Hergenhahn, U.; Schmidt, Ph.; Reiß, P.; Ozga, C.; Kambs, B.; Trinter, F.; Voigtsberger, J.; et al. Detecting Ultrafast Interatomic Electronic Processes in Media by Fluorescence. New J. Phys. 2014, 16, 102002.

(43) Viefhaus, J.; Scholz, F.; Deinert, S.; Glaser, L.; Ilchen, M.; Seltmann, J.; Walter, P.; Siewert, F. The Variable Polarization XUV Beamline P04 at PETRA III: Optics, Mechanics and their Performance. Nucl. Instrum. Methods Phys. Res., Sect. A 2013, 710, 151-154. 
(44) Winter, B.; Hergenhahn, U.; Faubel, M.; Björneholm, O.; Hertel, I. V. Hydrogen Bonding in Liquid Water Probed by Resonant Auger-Electron Spectroscopy. J. Chem. Phys. 2007, 127, 094501.

(45) Schirmer, J.; Trofimov, A. B.; Randall, K. J.; Feldhaus, J.; Bradshaw, A. M.; Ma, Y.; Chen, C. T.; Sette, F. K-Shell Excitation of the Water, Ammonia, and Methane Molecules Using High-Resolution Photoabsorption Spectroscopy. Phys. Rev. A 1993, 47, 1136-1147.

(46) Wenåker, I. The Spectrum of Singly Ionized Oxygen, O II. Phys. Scr. 1990, 42, 667-684.

(47) Knie, A.; Burbank, N.; Schmidt, P.; Ozga, C.; Ehresmann, A. Electron-Impact Induced Fluorescence for EUV Spectrometer-Detector Calibration. J. Electron Spectrosc. Relat. Phenom. 2012, 185, 492-497.

(48) Smith, J. W.; Lam, R. K.; Shih, O.; Rizzuto, A. M.; Prendergast, D.; Saykally, R. J. Properties of Aqueous Nitrate and Nitrite from X-ray Absorption Spectroscopy. J. Chem. Phys. 2015, 143, 084503.

(49) Heller Jr., J. M.; Hamm, R. N.; Birkoff, R. D.; Painter, L. R. Collective Oscillation in Liquid Water. J. Chem. Phys. 1974, 60, 3483-3486.

(50) Winter, B.; Weber, R.; Widdra, W.; Dittmar, M.; Faubel, M.; Hertel, I. V. Full Valence Band Photoemission from Liquid Water Using EUV Synchrotron Radiation. J. Phys. Chem. A 2004, 108, 2625-2632.

(51) German, K. R. Direct Measurement of the Radiative Lifetimes of the $\mathrm{A}^{2} \Sigma^{+}\left(V^{\prime}=0\right)$ States of OH and OD. J. Chem. Phys. 1975, 62, 2584

(52) Carpenter, C. M.; Sun, C.; Pratx, G.; Rao, R.; Xing, L. Hybrid X-ray/Optical Luminescence Imaging: Characterization of Experimental Conditions. Med. Phys. 2010, 37, 4011-4018. 
(53) Chen, H.; Longfield, D. E.; Varahagiri, V. S.; Nguyen, K. T.; Patrick, A. L.; Qian, H.; VanDerveer, D. G.; Anker, J. N. Optical Imaging in Tissue with X-ray Excited Luminescent Sensors. Analyst 2011, 136, 3438-3445. 
Graphical TOC Entry

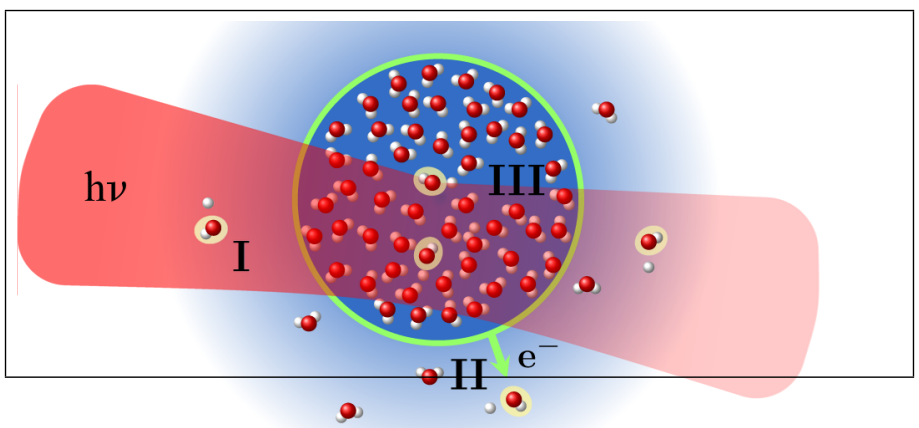

